vol. $21-n^{\circ} 3 \mid 2005$

Familles, destins personnels et appartenances

collectives en migration

\title{
Familles, destins personnels et appartenances collectives en migration
}

Catherine Delcroix et Lamia Missaoui

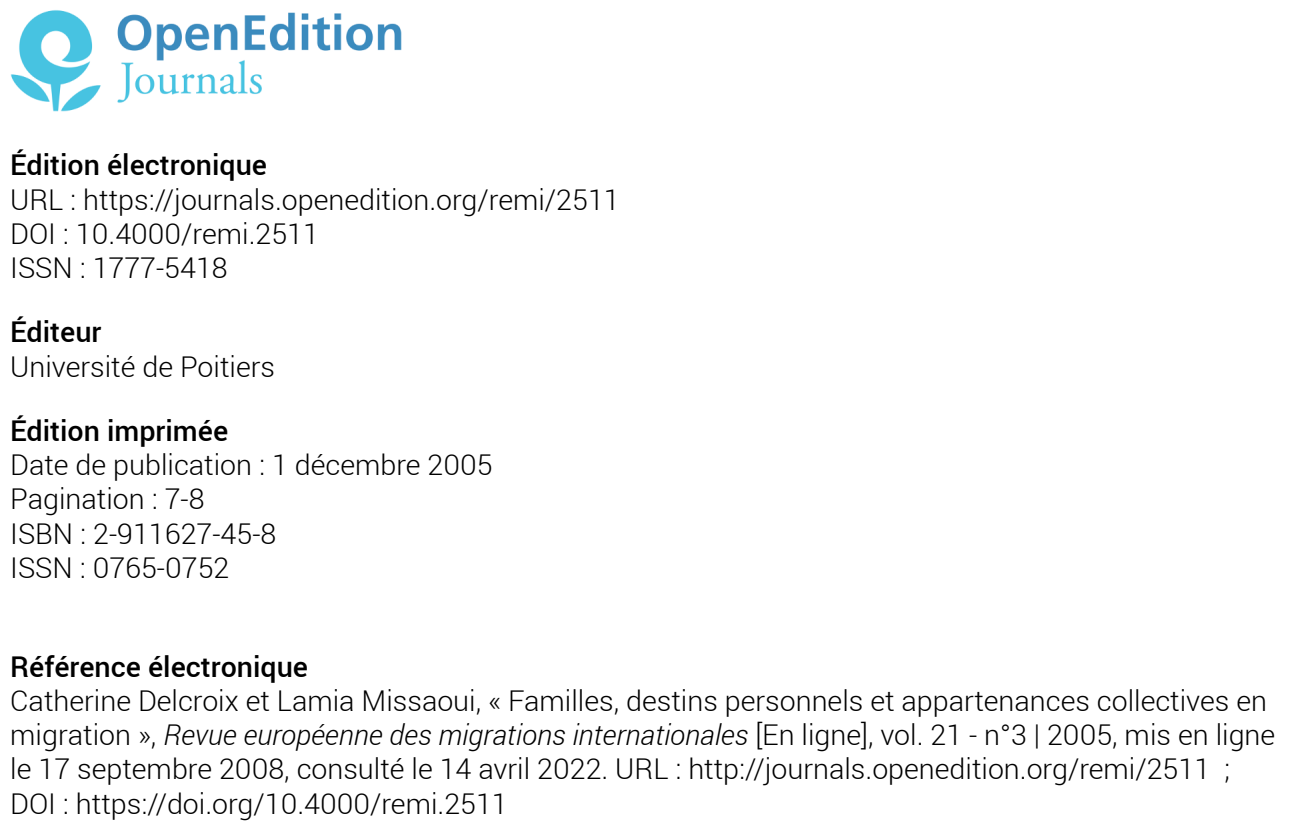

Ce document a été généré automatiquement le 14 avril 2022.

(c) Université de Poitiers 


\title{
Familles, destins personnels et appartenances collectives en migration
}

\author{
Catherine Delcroix et Lamia Missaoui
}

1 Ce dossier présente trois articles et deux notes de recherche consacrés aux activités par lesquelles des migrants forment leurs destinées et celles de leurs enfants, notamment en fonction de leurs ressources familiales. L'accent est mis sur les liens émotionnels, culturels et économiques que des familles et des individus en migration tissent entre leur lieu d'origine et leurs lieux d'installation. Ces liens sont étudiés à diverses échelles : quartier, ville, pays et continent ${ }^{1}$.

2 Comment se confrontent les modèles familiaux traditionnels et ceux de la société d'accueil ? Tradition et modernité sont-elles en opposition ou constituent-elles deux ensembles au sein desquels les migrants puisent alternativement des ressources en fonction de leurs stratégies propres, dans un effort de bricolage constructif? Comment font-ils pour faire face aux déséquilibres engendrés par la précarité de leur situation économique, administrative, politique (racisme, participation) et sociale? Quels rôles jouent les différents contextes institutionnels locaux et nationaux (Grande-Bretagne, Allemagne, France) sur les chances de vie et la construction des destins individuels de ces femmes et de ces hommes.

3 Tous les textes du dossier présentent des phénomènes et processus sociologiques étudiés à partir d'études de cas de familles et de récits de vie. Les historiques de famille prolongent la méthode biographique, qui cherche à reconstruire des histoires individuelles tout en les réinsérant dans leurs contextes micro et méso-sociaux. Étendre la mise en œuvre de la méthode à tout un groupe familial, et multiplier les « histoires de cas » de familles partant de conditions similaires, permet d'appréhender les dynamiques intergénérationnelles et de genre internes à ces groupes. Les réinsérer dans les contextes locaux de la société d'accueil (l'école, le quartier, les réseaux de sociabilités, la rue, les marchés du travail...) permet de comprendre comment ces contextes fonctionnent à l'égard des migrants et de leurs enfants. La similarité 
méthodologique ouvre une perspective de comparaison internationale. Des traits communs se dégagent-ils entre les stratégies de migrants turcs en Allemagne, Bengalis en Grande-Bretagne, Jamaïcains en Grande-Bretagne mais aussi aux États-Unis et au Canada, Chinois en France et femmes maliennes et indiennes en France?

4 Paul Thompson et Elaine Bauer décrivent un modèle de famille étendue matrifocale, solidaire et pragmatique, caractéristique de la migration jamaïcaine et en soulignent la grande modernité.

5 Kate Gavron pour les Bengalis émigrés à Londres et Ursula Apitzsch pour les Turcs en Allemagne traitent toutes les deux de l'ethnic business. L'une et l'autre montrent que si la petite entreprise familiale constitue une solution, parfois la seule, d'insertion économique, elle présente de lourds inconvénients pour les fils des migrants tenus d'abandonner très tôt leurs études pour travailler dans la micro entreprise tandis que leurs sœurs peuvent continuer à étudier. Kate Gavron décrit des stratégies matrimoniales originales mises en œuvre par des jeunes Bengalies de Londres qui combinent à leur profit modernité et tradition.

Chloé Cattelain, Marylène Lieber, Claire Saillard et Sébastien Ngugen montrent comment des parents et particulièrement des femmes d'une quarantaine d'années vivant dans des zones de Chine du Nord sinistrées par la fermeture de très grandes usines d'État émigrent dans un pays occidental en l'occurrence en France... Ils n'ont pas d'espoir de retrouver un emploi dans des entreprises privées de leur pays faute d'avoir $\mathrm{pu}$ se former pendant la révolution culturelle. De plus ils se trouvent dans l'impossibilité d'offrir à leurs enfants les moyens de se construire un meilleur avenir. C'est à cause de ce contexte difficile qu'ils font le choix coûteux et périlleux de venir travailler comme clandestins en France.

Enfin Blandine Veith étudie le cas de deux femmes : une Malienne et une Indienne, immigrées en France. Toutes deux sont engagées dans un processus d'individuation qui, souligne l'auteur a commencé avant leur émigration. En France, elles s'appuient sur des collectifs multiculturels de femmes pour trouver un équilibre entre leur appartenance communautaire et leur volonté d'autonomie.

\section{NOTES}

1. L'ensemble de ce dossier est issu d'une journée d'étude internationale organisée en avril 2003 sur le thème des "résistances des familles immigrées à la précarité de leur situation" par le Pôle «Institutions» du laboratoire Printemps (Professions, Institutions, Temporalités) à l'Université de Versailles Saint-Quentin en Yvelines. 
INDEX

Mots-clés : appartenance, relations avec le pays d'origine, relations familiales

\section{AUTEURS}

\section{CATHERINE DELCROIX}

Maître de Conférences, HDR en sociologie, Laboratoire Printemps, Université de Saint-Quentin en Yvelines, 47, bld Vauban 78047 Guyancourt, caldelcroix@wanadoo.fr

\section{LAMIA MISSAOUI}

Maître de Conférences, Laboratoire Printemps, Université de Saint-Quentin en Yvelines, 47, bld Vauban 78047 Guyancourt, lamia.missoui@libertysurf.fr 